\title{
Metodologia para avaliação ultrassonográfica da biometria uterina na fêmea bovina
}

\section{Methodology for ultrasound evaluation of uterine biometry in the bovine female}

\author{
Metodología para la evaluación ultrasonográfica de la biometría uterina en la \\ hembra bovina
}

Gómez-L Víctor ${ }^{1}$ M.Sc; Pereira Jhonata ${ }^{1}$ M.Sc; Okano Denise ${ }^{1}$ M.Sc; Dos Santos Giancarlo² Ph.D; Marcondes Marco ${ }^{3}$ Ph.D; Benjamin Laercio ${ }^{1}$ Ph.D; Guimarães Jose ${ }^{1}$ Ph.D; Ramírez-L Camilo ${ }^{1 *}$ M.Sc.

${ }^{1}$ Universidade Federal de Viçosa, Departamento de Medicina Veterinária, avenida PH Rolfs, Viçosa, Brasil.

2 UNIVIÇOSA, Escolha de Ciências Biológicas e da Saúde FACISA, Avenida Castelo Branco, Viçosa, Brasil.

${ }^{3}$ Universidade Federal de Viçosa, Departamento de Zootecnia, avenida PH Rolfs, Viçosa, Brasil. Brasil.

Palavras chaves:

Diâmetro dos cornos uterinos; espessura endometrial; fertilidade;

holstein.

\section{Key words:}

Diameter of the uterine horn; endometrial thickness; fertility; holstein.

INFORMACIÓN

Recibido: 29-01-2017; Aceptado: 05-06-2017. *Correspondencia autor: camilo2407@gmail.com

\section{Resumo}

Objetivou-se avaliar uma metodologia para mensurar a espessura endometrial e diâmetro dos cornos uterinos. Para o qual, os cornos uterinos foram divididos em três regiões. A partir do septo intercornual até o início a curvatura maior, foi considerada a primeira região. A partir da curvatura maior até o início do ápice do corno segunda região, e extremidade livre do corna a terceira região. Mensurou-se a espessura endometrial e o diâmetro dos cornos em animais in vivo e nos órgãos genitais dos mesmos post mortem. Foram utilizadas cinco vacas sexualmente maturas da raça Holandesa, criadas em manejo Losing-House. Os dados quantitativos foram analisados pela ANOVAe as médias comparadas pelos testes de Tukey ou pela análise não paramétrica comparando as médias pelo teste de Kruskall Wallis. Os valores médios não apresentaram diferenças para as características avaliadas no animal in vivo comparadas aos obtidos post mortem $(P>0,05)$. Nas mensurações obtidas, a terceira região mostrou valores médios semelhantes para diâmetro $(21,0 \pm 2,6 \mathrm{~mm}-22,9 \pm 2,8 \mathrm{~mm})$ e espessura endometrial de ambos cornos $(10,4 \pm 4,9 \mathrm{~mm}-10,8 \pm 5,2 \mathrm{~mm})$. De igual forma os valores obtidos para espessura e diâmetro de ambos cornos uterinos da terceira região mostrou-se semelhante aos valores obtidos na segunda e primeira região. Já os valores da segunda região diferiram dos obtidos na primeira região $(P<0,05)$. Dessa forma, a obtenção de imagens ultrassonográficas em cortes transversais e a mensuração das biometrias uterinas a partir da bifurcação até a curvatura maior, mostra-se eficiente e representativa da espessura endometrial e diâmetro dos cornos uterinos como um tudo.

\begin{abstract}
The objective was to evaluate a methodology to measure the endometrial thickness and diameter of the uterine horns. For which, the uterine horns were divided into three regions. From the intercornual septum to the beginning the greater curvature, it was considered the first region. From the greatest curvature to apex of the second horn region, and free end of the horn the third region. The endometrial thickness and the diameter of the horns were measured in animals in vivo and in the genital organs of the same post mortem. Five sexually mature cows of the Holstein breed, raised in Losing-House management, were used. The quantitative data were analyzed by ANOVA and the means compared by Tukey's tests or by non-parametric analysis comparing the means by the Kruskall Wallis test. The mean values did not present differences for the characteristics evaluated in the animal in vivo compared to those
\end{abstract}


obtained post mortem $(P>0.05)$. In the measurements obtained, the third region showed similar mean values for diameter $(21.0 \pm 2.6 \mathrm{~mm}-22.9 \pm 2.8 \mathrm{~mm})$ and endometrial thickness of both horns $(10.4 \pm 4.9 \mathrm{~mm}-10,8 \pm 5.2 \mathrm{~mm})$. Likewise, the values obtained for thickness and diameter of both uterine horns of the third region was similar to the values obtained in the second and first regions. However, the values of the second region differed from those obtained in the first region ( $P$ $<0.05)$. Thus, obtaining ultrasound images in cross-sections and measuring uterine biometrics from bifurcation to greater curvature, is efficient and representative of the endometrial thickness and diameter of the uterine horns as a whole.

\section{Palabras Clave:}

Diámetro de los cuernos uterinos; espesura endometrial; fertilidad; holstein

\section{Resumen}

El objetivo de este estudio fue evaluar una metodología para medir la espesura endometrial y el diámetro de los cuernos uterinos. Para lo cual, los cuernos uterinos fueron divididos en tres regiones. A partir del septo intercornual hasta el inicio de la curvatura mayor, fue considerada la primera región; a partir de la curvatura mayor hasta el inicio del ápice del cuerno, la segunda región, y la extremidad libre del cuerno la tercera región. Se midió la espesura endometrial y el diámetro de los cuernos uterinos en animales in vivo y en los órganos genitales de los mismos animales post mortem. Fueron utilizadas cinco vacas sexualmente maduras de la raza Holstein criadas en manejo Losing-House. Los datos cuantitativos fueron analizados por ANOVA y las medidas comparadas por los testes de Tukey o por análisis no paramétricos comparando las medias por el teste de Kruskall Wallis. Los valores medios no presentaron diferencias entre las características evaluadas en los animales in vivo comparadas a las obtenidas post mortem $(P>0,05)$. En las medidas obtenidas, la tercera región mostro valores medios semejantes para diámetro $(21,0 \pm 2,6 \mathrm{~mm}-22,9 \pm 2,8 \mathrm{~mm})$ y espesura endometrial de ambos cuerno cornos $(10,4 \pm 4,9 \mathrm{~mm}-10,8 \pm 5,2 \mathrm{~mm})$. De igual forma los valores obtenidos para espesura y diámetro de ambos cuernos uterinos de la tercera región se mostro semejante a los valores obtenidos en la segunda y primera región. Ya los valores de la segunda región difirieron de los obtenidos en la primero región $(P<0,05)$. De esa forma, la obtención de imágenes ecográficas en cortes transversales y las medidas de las biometrías uterinas a partir de la bifurcación hasta la curvatura mayor, se mostraron eficientes y representativas de la espesura endometrial y diámetro de los cuernos uterinos como un todo.

\section{Introdução}

A literatura geralmente relata o eixo hipotálamohipofisário-gonadal como o responsável pelo controle e desenvolvimento do ciclo estral, deixando de lado o próprio útero, o qual é um órgão essencial para a procriação e perpetuação das espécies. Sendo que, exerce parte essencial na modulação do ciclo estral por meio de mecanismos luteolíticos das células endometriais. Além de atuar no transporte e capacitação dos espermatozoides, bem como na implantação, desenvolvimento, alojamento, nutrição e proteção do conceito até o final da gestação (HAFEZ e HAFEZ, 2004). Possui também função especial no parto e na expulsão das membranas placentárias, para a recuperação dos tecidos com finalidade de nova gestação (ALMEIDA et a., 2013).

Em humanos, a espessura endometrial é bem definida na literatura, de forma que é possível identificar endometrite ou mesmo a sua relação com fertilidade. Diversos estudos têm sido realizados em bovinos, no intuito de avaliar o grau de maturidade sexual de fêmeas aptas à reprodução (ANDERSON et al. 1991; HOLM et al., 2009). Porém, na literatura consultada não foi encontrada a descrição de uma metodologia exata para avaliar a biometria uterina nesta espécie. Da mesma forma, a ultrassonografia tem sido empregada na avaliação da camada endometrial, com a finalidade de correlaciona-la com fertilidade (PIERSON e GINTHER, 1987; MONA et al., 2014). Ou mesmo na avaliação da involução uterina e o diagnostico de infecções uterinas em ausência de liquido (POLAT et al., 2015). Neste sentido objetivou-se no presente estudo, padronizar e descrever uma metodologia de alta acurácia por meio da obtenção e interpretação de imagens ultrassonográficas, que permita mensurar a espessura endometrial e o diâmetro dos cornos uterinos em bovinos. 


\section{Material e métodos}

Local: O presente estudo foi conduzido com animais da Unidade de Ensino, Pesquisa e Extensão em Gado de Leite (UEPE-GL) do Departamento de Zootecnia (DZO) da Universidade Federal de Viçosa (UFV) localizada a $-20^{\circ} 45^{\prime} 16^{\prime \prime}$ latitude norte e $42^{\circ} 52^{\prime} 57^{\prime \prime}$ longitude leste no estado de Minas Gerais. A uma altura de 660 metros acima do nível do mar, com clima tropical, chuvas durante o verão, e temperatura entre $10^{\circ} \mathrm{C}$ e $23^{\circ} \mathrm{C}$, com média de $19^{\circ} \mathrm{C}$, (CARDONA et al., 2013). O presente trabalho foi executado, sob o protocolo de aprovação No 88/2013 da comissão de Ética no Uso de AnimaisCEUA da UFV.

Animais: Foram utilizadas cinco fêmeas bovinas adultas da raça Holandesa, cíclicas e sexualmente maduras, da ordem de parto igual a quatro, as quais se encontravam clinicamente hígidas, livres de patologias nos órgãos genitais. Indicadas ao abate por critérios zootécnicos e estratégia de reposição do rebanho de acordo com o manejo de produção da UEPL do DZO-UFV. As fêmeas foram mantidas em sistemas estabulados Losing-House, em baias coletivas $\left(12 \mathrm{~m}^{2} /\right.$ animal) de acordo com a produção leiteira, alimentadas com ração e silagem de milho fornecidos duas vezes ao dia logo após as ordenhas (7:30 e 16:30 horas). Suplemento mineral proteínado e água foram fornecidos para consumo ad-libitum. O estábulo possui sistema de ventilação para a dissipação do calor, camas individuais secas e limpas.

Espessura endometrial e diâmetro dos cornos uterinos: Para facilitar a mensuração da espessura endometrial e diâmetro dos cornos uterinos, o útero foi dividido em três regiões, modificando os segmentos preconizados por Ginther (1998). A primeira região compreendeu a zona do ápice do corno uterino. A segunda região foi considerada a partir do ápice até a curvatura maior. E finalmente, a terceira região desde a curvatura maior até o início da bifurcação interna dos cornos uterinos (ainda unidos pelo septo intercornual). Foi utilizado transdutor em frequência 7,5 MHz (Mindray ${ }^{\circledR} 75$ L50EAV) acoplado ao ultrassom Mindray ${ }^{\circledR}$ Dp2200.

O transdutor foi posicionado a $90^{\circ}$ de inclinação, e perpendicularmente em relação ao corno de tal forma que fosse obtido a maior superfície de contato sem chegar a deformar o corno evitando erros que gerassem imagens fora do padrão comum da anatomia dos órgãos genitais. Imagens ultrassonográficas foram congeladas em cada uma das regiões estabelecidas para mensuração da biometria uterina. A espessura endometrial foi considerada como camada visualizada desde o lume uterino até a interface do miométrio (Souza et al 2011). Para maior acurácia, foi obtida através da média das mensurações da camada endometrial dorsal e ventral. Medidas discrepantes em mais de um milímetro entre as duas camadas foram refeitas. O diâmetro do corno foi obtido pela média de duas mensurações perpendiculares ao diâmetro do útero.
Mensurações in vivo: Doze horas antes do abate foram feitos exames dos órgãos genitais de cada um dos animais. As vacas foram contidas em tronco individual, em seguida, foi feita a limpeza do reto e introduzido o transdutor transretal linear. Foram obtidas e salvas imagens ultrassonográficas para posteriores mensurações da espessura endometrial e o diâmetro uterino em cada uma das três regiões uterinas estabelecidas.

Mensurações post mortem: Os animais foram abatidos no frigorifico experimental do Departamento da Zootecnia (DZO-UFV). Após o abate, foram colhidos os órgãos genitais, e refrigerados em gelo reciclável dentro de caixas isotérmicas até o Laboratório de Anatomia Animal (DVT/UFV). Posteriormente, cada peça foi colocada dentro de cubas cheias de água, para a obtenção das mensurações da biometria nas três regiões uterinas por meio de ultrassonografia. Logo, cada corno foi seccionado transversalmente em cada região proposta para identificação das camadas uterinas e interpretação das imagens ultrassonográficas.

Análise estatística: Todos os dados foram submetidos à estatística descritiva com obtenção das médias, desvios-padrão e coeficiente de variação. Os dados quantitativos foram submetidos aos testes de Lillierfors e de Cochran e Bartlett, para verificar respectivamente, a normalidade dos dados e a homogeneidade das variâncias. A análise de variância (ANOVA) foi usada para avaliações de todos os dados que atenderem às premissas da ANOVA. Os testes de Tukey ou Duncan foram empregados para comparação das médias e quando os dados não atenderam as premissas da ANOVA (normalidade dos dados e homogeneidade das variâncias), os dados foram avaliados por meio de análise não paramétrica e as médias comparadas pelos testes de Kruskall Wallis ou Wilcoxon, empregando-se $5 \%$ de probabilidade de erro. Quando os valores de variação foram superiores a $15 \%$, empregou-se o teste de Duncan, no intuito de evitar o erro estatístico tipo II. Correlações simples de Pearson foram realizadas entre todas as características estudadas.

\section{Resultados}

Os valores médios obtidos a partir das imagens ultrassonográficas nos animais in vivo e posteriormente nas mensurações post mortem da espessura endometrial e do diâmetro uterino estão sumarizados na Tabela 1. Não foram observadas diferenças $(p>0,05)$ entre as medias das espessuras endometriais e diâmetro uterino dos cornos esquerdos e direito antes e depois do abate.

Os valores médios por região para espessura endometrial e o diâmetro do corno esquerdo e direito nos animais são apresentados na Tabela 2. Observa-se que os valores da espessura endometrial e diâmetro uterino post mortem mostram-se superiores nas regiões dois e três ao se comparar com a primeira região. As medias da primeira e terceira a região para a variável 
espessura endometrial dos cornos uterinos são semelhantes entre elas, mas inferiores ao comparar-se com os valores da região dois.

Tabela 1. Valores médios e desvio padrão da espessura endometrial e o diâmetro uterino $(\mathrm{mm})$ in vivo e post mortem de vacas da raça Holandesa, criadas em regime Losing-House.

\begin{tabular}{cccc}
\hline Caracteristicas & $\boldsymbol{N}$ & $\boldsymbol{P M}$ & MÉDIA \\
\hline EECE & $10,6 \pm 4,7$ & $11,5 \pm 4,9$ & $10,9 \pm 4,9$ \\
DCE & $20,5 \pm 3,6$ & $23,4 \pm 4,9$ & $21,7 \pm 4,2$ \\
EECD & $10,5 \pm 4,6$ & $11,0 \pm 4,7$ & $10,6 \pm 4,6$ \\
DCD & $22,8 \pm 4,3$ & $23,7 \pm 3,9$ & $23,0 \pm 3,9$ \\
\hline
\end{tabular}

$(P>0,05)$ na mesma linha, pelo teste de Tukey; IV: in vivo; PM: post mortem; EECE: Espessura endometrial corno esquerdo; DCE: Diâmetro corno esquerdo; EECD: Espessura endometrial corno esquerdo; DCD: Diâmetro corno direito.

Tabela 2. Valores médios e desvio padrão da espessura endometrial e diâmetro do corno uterino $(\mathrm{mm})$ in vivo e post mortem de acordo com a região do corno uterino de vacas da raça Holandesa criadas em regime Losing-House.

\begin{tabular}{cccc}
\hline Características & In Vivo & Post Mortem & Média \\
\hline \multicolumn{4}{c}{ Primeira região } \\
EECE & $10,0 \pm 4,6$ & $9,9 \pm 5,2$ & $9,6 \pm 4,8$ \\
DCE & $17,1 \pm 2,7$ & $19,1 \pm 2,2$ & $18,2 \pm 2,5$ \\
EECD & $9,3 \pm 4,3$ & $8,9 \pm 3,7$ & $8,5 \pm 3,6$ \\
DCD & $19,0 \pm 3,6$ & $19,8 \pm 2,3$ & $19,5 \pm 2,8$ \\
& Segunda região & \\
EECE & $11,6 \pm 5,5$ & $13,3 \pm 4,7$ & $12,3 \pm 4,9$ \\
DCE & $22,9 \pm 3,7$ & $26,8 \pm 4,7$ & $24,2 \pm 3,9$ \\
EECD & $11,4 \pm 5,0$ & $12,9 \pm 5,4$ & $12,7 \pm 4,9$ \\
DCD & $25,6 \pm 3,9$ & $26,8 \pm 2,7$ & $25,8 \pm 3,3$ \\
& Terceira região & \\
EECE & $10,4 \pm 4,9$ & $11,7 \pm 5,4$ & $10,7 \pm 5,1$ \\
DCE & $21,0 \pm 2,6$ & $24,2 \pm 4,2$ & $22,8 \pm 3,8$ \\
EECD & $10,8 \pm 5,2$ & $11,3 \pm 4,8$ & $10,6 \pm 4,8$ \\
DCD & $22,9 \pm 2,8$ & $24,4 \pm 3,0$ & $23,7 \pm 2,8$
\end{tabular}

$(P>0,05)$ na mesma linha, pelo teste de Tukey; EECE: Espessura endometrial corno esquerdo; DCE: Diâmetro corno esquerdo; EECD: Espessura endometrial corno esquerdo; DCD: Diâmetro corno direito.

Os valores da Tabela 3 representam a comparação por regiões, indicando que o diâmetro dos cornos uterinos e a espessura endometrial na terceira região mostrara-se semelhante aos valores médios das outras regiões mensuradas $(P>0,05)$. Porém, os valores médios da primeira região para o diâmetro do corno uterino esquerdo mostraram valores inferiores aos da segunda região $(P<0,05)$.

Tabela 3. Valores médios e desvio padrão $(\mathrm{mm}) \mathrm{da}$ espessura endometrial e diâmetro dos cornos uterinos in vivo, comparados por região em vacas da raça Holandesa criadas em regime Losing-House.

\begin{tabular}{ccccc}
\hline Características & Primeira região & Segunda região & Terceira região & Média \\
\hline EECE & $9,9 \pm 4,6$ & $11,6 \pm 5,5$ & $10,4 \pm 4,9$ & $10,2 \pm 4,9$ \\
DCE & $17,1 \pm 2,7 \mathrm{~B}$ & $22,9 \pm 3,2 \mathrm{~A}$ & $21,0 \pm 2,6 \mathrm{AB}$ & $20,5 \pm 3,6$ \\
EECD & $9,3 \pm 4,3$ & $11,4 \pm 5,0$ & $10,8 \pm 5,2$ & $9,9 \pm 4,7$ \\
DCD & $19,0 \pm 3,6$ & $25,6 \pm 3,9$ & $22,9 \pm 2,8$ & $22,7 \pm 4,3$ \\
\hline
\end{tabular}

Valores médios seguidos por letras maiúsculas diferentes na mesma linha diferem entre si $(P<0,05)$ pelo teste de Tukey; EECE: Espessura endometrial corno esquerdo; DCE: Diâmetro corno esquerdo; EECD: Espessura endometrial corno esquerdo; DCD: Diâmetro corno direito.

\section{Discussão}

A ultrassonografia vem sendo utilizada cada vez mais como uma ferramenta auxiliar ao exame dos órgãos genitais em bovinos (CORREDOR-CAMARGO e PAÉZ, 2012). PIERSON e GINTHER (1987) fizeram um dos primeiros relatos de ultrassonografia uterina em bovinos. Através de cortes longitudinais 15 milímetros após a cérvix os autores escanearam os cornos uterinos em sentido cranial. Porém, os mesmos relataram que nem sempre foram diferenciadas claramente as camadas uterinas nas imagens ultrassonográficas. Além disso, a técnica utilizada não permitiu o aproveitamento da imagem gerada para mensurar o diâmetro uterino.

Com o avanço dos aparelhos ultrassonográficos e sua precisão, novos estudos ultrassonográficos reportam a possibilidade de diferenciar as camadas uterinas (SOUZA et al., 2011; POLAT et al., 2015). A literatura consultada sobre ultrassonografia em bovinos, não mostra em detalhe a delimitação das camadas uterinas o que dificulta a interpretação das imagens e a relação da espessura endometrial com fertilidade ou diagnostico de patologias uterinas como endometrite em ausência de liquido no lume, como é feito em mulheres.

Dessa forma, a interpretação das imagens obtidas por ultrassonografia, só ficou esclarecida após revisão e associação com a histologia uterina relatada por DELLMANN e BROWN (1982). Assim, foi possível identificar e delimitar as camadas uterinas por meio do presente estudo (Fig. 1). Sendo que, a camada mais interna das imagens é formada pelo endométrio seguido de uma fina camada muscular transversal. Posteriormente, delimitado por um tecido frouxo ricamente vascularizado (estrato vascular que gera uma imagem anecóica) e finalmente uma camada muscular longitudinal em união ao perimétrio.

Sabendo que as medidas obtidas no animal in vivo são acuradas (Tabela 1 e 2), o seguinte passo foi padronizar o local de mensuração dos cornos uterinos. A terceira região uterina apresentou valores médios e semelhantes às outras duas regiões. Dessa forma, a mesma, mostra-se representativa do útero como um todo para obter medidas da espessura endometrial e diâmetro dos cortes uterinos por meio de cortes transversais de imagens ultrassonograficas.

DESCÔTEAUX et al.(2010) coincide com BARLUND et al., (2008) ao relatar que a aparência do endométrio pode mudar de acordo à idade e fase do ciclo em que seja feita o exame. No entanto os primeiros não relataram uma metodologia para obter dita mensuração. Já os segundos utilizaram três imagens diferentes do útero, em cortes transversais para determinar a espessura endometrial, sem descrever em quais locais foram obtidas ditas imagens.

Caso parecido aconteceu com o trabalho de POLAT et al. (2015), os quais relataram a obtenção de três imagens na curvatura maior do útero para obter uma 


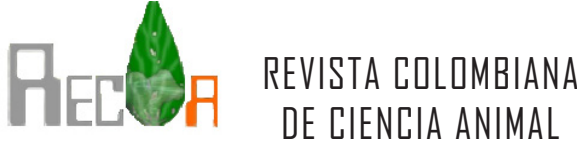
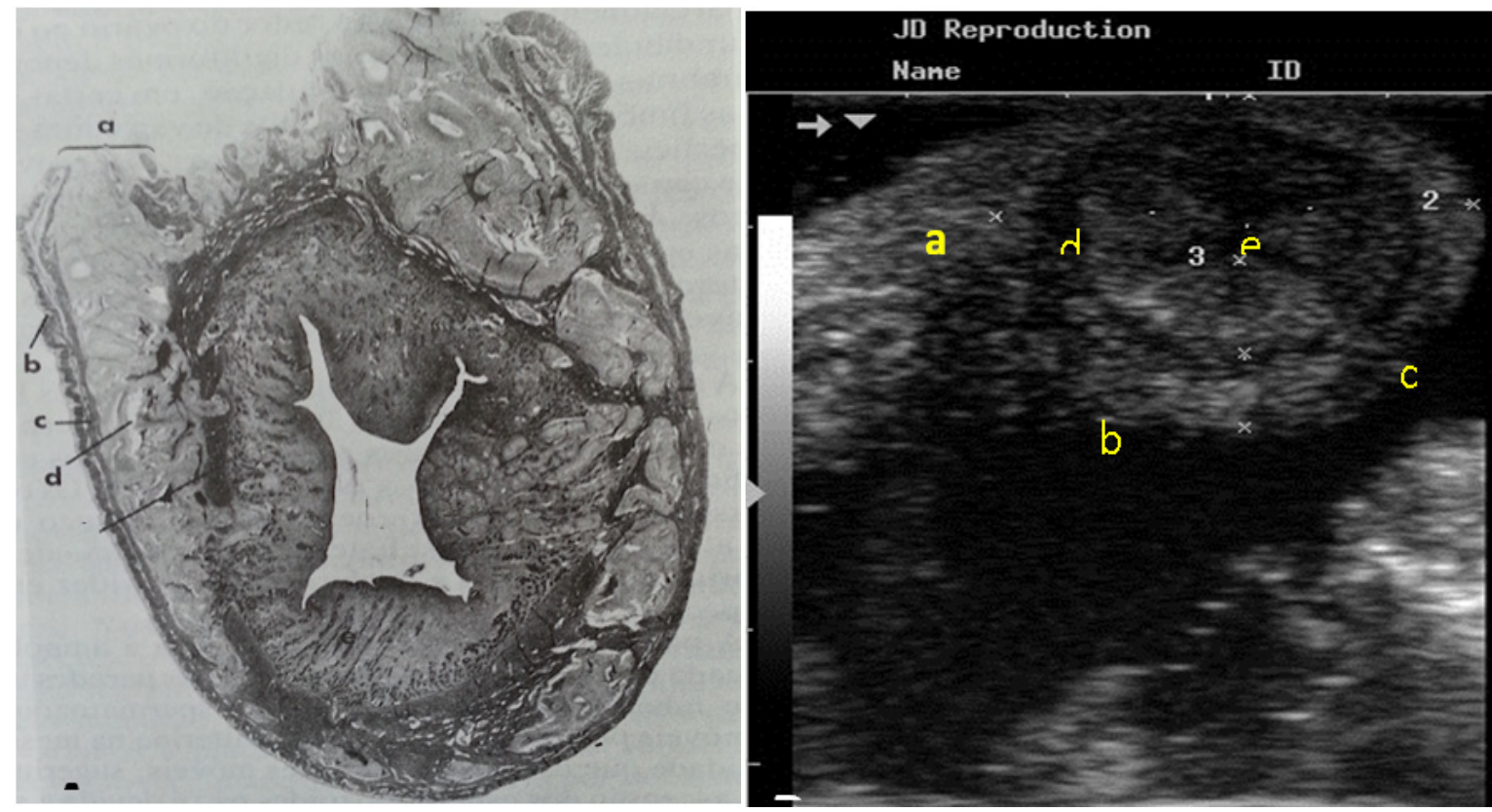

Figura 1. Aspecto histológico (A) e ultrassonográfico (B) do ligamento largo do útero (a), das camadas serosas (b), muscular longitudinal (c), e vascular (d), e do lume do útero (é). Fonte: A: Dellmann e Brown (1982); B: Arquivo pessoal.

maior acurácia da espessura endometrial do corno. No entanto, de acordo com os dados obtidos no presente estudo, a curvatura maior do corno esquerdo (segunda região) não foi semelhante com espessura e diâmetro do ápice do mesmo corno (primeira região). Não representando a biometria uterina como um tudo. Contrastando com a metodologia utilizada por SOUZA et al. (2011), os quais obtiveram imagens transversais em sentido cranial à bifurcação uterina. Estes autores, não relatam o motivo da escolha desses locais. Os dados obtidos no presente estudo constatam que só a terceira região do útero seria representativa das outras regiões.

MONTEIRO et al. (2013), mensuraram a espessura endometrial de um só corno. Porém, as estruturas no ovário poderiam influenciar mais o corno ipsilateral, podendo haver uma mudança maior acima dele, de forma que a hipótese deveria ser testada em um maior numero de animais.

O diâmetro obtido dos cornos uterinos obtidos no presente trabalho, não coincidem com os valores preconizados por ANDERSON et al. (1991), onde as fêmeas maturas sexualmente aptas à reprodução com devem apresentar diâmetro de cornos uterinos superior a 30 milímetros. Por outro lado, os diâmetros uterinos obtidos neste estudo coincidem com os valores médios propostos por MIHURA e CASARO (1999), os quais modificaram os valores preconizados por ANDERSON (1991), e relataram diâmetros uterinos de $20 \mathrm{~mm}$ para úteros de animais maduros sexualmente. Contudo, têm que ressaltar que estes valores foram preconizados para novilhas, e no presente estudo foram utilizadas fêmeas adultas pluríparas. Para dita comparação, sugere-se a obtenção da biometria uterina em animais desde a fase peri puberal até sua maturidade sexual.

No entanto, a cavidade pélvica em novilhas normalmente é estreita o que poderia dificultar a movimentação da mão juntamente com o transdutor para gerar as imagens de cortes transversais. Indica-se então, girar o transdutor na palma da mão, ou posicionar o corno transversalmente antes de posicionar o transdutor. Erros de posicionamento e/ou pressão excessiva nos cornos são detectados na imagem gerada no ultrassom. O sucesso da metodologia mesmo se mostrando eficiente e atingindo sua finalidade, irá depender do treinamento do avaliador.

Considerando os estudos que relatam mudanças na espessura endometrial dos cornos uterinos ao longo do ciclo estral (PIERSON e GINTHER, 1987; SOUZA et al., 2011) e as correlações positivas entre o diâmetro uterino e o ganho de peso, idade e peso corporal, puberdade e maturidade sexual relatadas por MONTANHOLI et al. (2004), e ROBSON et al. (2007), indica-se o uso da presente metodologia para o estudado da biometria uterina de forma associada em diferentes faixas etárias e ao longo do ciclo estral de fêmeas bovinas.

\section{Conclusões}

Os valores médios obtidos para as diferentes áreas dos cornos uterinos, no animal in vivo e nos órgãos colhidos post mortem, indicam que a ultrassonografia transretal bovina permite identificar com alta acurácia as camadas muscular, vascular e endometrial do corno uterino, 
assim como seu lume. Imagens transversais obtidas na terceira região uterina, apresentaram neste estudo, valores médios e semelhantes às demais regiões do útero para a biometria uterina, sendo assim, o ponto indicado para a obtenção desses valores.
Agradecimentos. Ao programa de estudante Convênio de Pós-Graduação (PEC-PG), ao Conselho Nacional de Desenvolvimento Cientifico e Tecnológico (CNPq), à Coordenação de Aperfeiçoamento de Pessoal de Nível Superior (CAPES) e à Fundação de Amparo à Pesquisa do Estado de Minas Gerais (FAPEMIG) pelo apoio e financiamento.

\section{Referências}

ALMEIDA, O.M.; PINHO, R.O.; LIMA, D.M.; MARTINS, L.F. 2013. Endocrinologia da puberdade em fêmeas bovinas. Revista Científica Eletrônica de Medicina Veterinária 11 (20):1-13.

ANDERSON, K.J.; LEFEVER, D.G.; BRINKS, J.S.; ADDE, K.G. 1991. The use of reproductive tract score in beef heifers. Agri-practice 12 (4): 19-26.

BARLUND, C.S.; CARRUTHERS, T.D.; WALDNER, C.L.; PALMER, C.W. 2008. A comparison of diagnostic techniques for postpartum endometritis in dairy cattle. Theriogenology 69:714-723.

CARDONA, J.A.; MONTES, J.C.; CASTAÑO, F.A.; BLANCO, R.D.; LEÓN, V.E. 2013. Frequência da dermatobiose cutânea bovina em vacas da raça Holandesa de uma granja leiteira de Viçosa (MG, Brasil). Revista CES Medicina Veterinaria y Zootecnia 8 (1):82-94.

CORREDOR-CAMARGO, E.S.; PAÉZ, E.M. 2012. Aplicaciones de la ultrasonografía en la reproducción bovina: revisión. Revista Ciencia y Agricultura 9 (2):29-37.

DELLMANN, H.D.; BROWN, E.M. 1982. Histologia veterinária. Guanabara, Rio de Janeiro. Brasil.

DESCÔTEAUX, L.; CHASTANT-MAILLARD, S.; GNEMMI, G.; COLLOTON, J.; BOLLWEIN, H. 2010. Practical Atlas of Ruminant and Camelid. Reproductive Ultrasonography Bovine Uterus. Université de Montréal, Canada.

GINTHER, O.J. 1998. Ultrasonic imaging and animal reproduction: Cattle, Book 3. Equiservices Publishing. Wisconsin.

HAFEZ, E.S.E.; HAFEZ, B.; 2004. Reprodução Animal. 7.ed. Manole, São Paulo. Brasil.

MIHURA, H.; CASARO, G. 1999. Selección de vaquillonas de reposición en rodeos de cría. Revista Taurus 1 (4):34-39.

MONA, J.; PAVANELO, J.R.; ALVES, L.; MEDEIROS, D.E.; CARVALHO, L.M.; PACHECO, G.; FERREIRA, R.M.; AYRES, H.; BARUSELLI, P.; PALMA, F.; PAPA, P.C. 2014. Treatment with eCG Decreases the Vascular Density and Increases the Glandular Density of the Bovine Uterus. Reprodução Domestica Animal 49:453-462.

MONTANHOLI, Y.R.; BARCELLOS, J.O.; BORGES, J.B.; DA COSTA, E.C.; WUNSH, C.; PRATES, E.R. 2004. Ganho de peso na recria e desempenho reprodutivo de novilhas acasaladas com sobreano. Pesquissa agropecuaria brasileira 39 (12):1253-1259.

MONTEIRO, F.M.; MERCADANTE, M.E.; BARROS, C.M.; SATRAPA, R.A.; SILVA, J.A.; OLIVEIRA, L.Z.; SARAIVA, N.Z.; OLIVEIRA, C.S.; GARCIA, J.M. 2013. Reproductive tract development and puberty in two lines of Nellore heifers selected for postweaning weight. Theriogenology 1 (8):10-17. 
POLAT, B.; CENGIZ, M.; CANNAZIK, O.; COLAR, A.; ORUC, E.; ALTUN, S.; SALAR, S.; BASTAN, A. 2015. Endometrial echotexture variables in postpartum cows with subclinical endometritis. Animal Reproduction Science 155:50-55.

PIERSON, R.A.; GINTHER, O.J. 1987. Ultrasonographic appearence of the bovine uterus during the estrous cycle. Journal of the American Veterinary Medical Association 190 (8):995-1001.

ROBSON, C.; MAGLIETTI, C.; LÓPEZ, S.; VOGEL, O.; CELSER, 2007. Grado de desarrollo reproductivo (gdr): su utilización en vaquillas entoradas a los 18 meses. Corrientes, Noticias y Comentarios 417:1-4.

SOUZA, A.H.; SILVA, E.P.; CUNHA, A.P.; GÜMEN, A.; AYRES, H.; BRUSVEEN, D.J.; GUENTHER, J.N.; WILTBANK, M.C. 2011. Ultrasonographic evaluation of endometrial thickness near timed Al as a predictor of fertility in highproducing dairy cows. Theriogenology 75:722-733. 